\title{
Thrombospondin-1 Deficiency Causes a Shift from Fibroproliferative to Inflammatory Kidney Disease and Delays Onset of Renal Failure
}

\author{
Michael Zeisberg, ${ }^{* \dagger}$ Björn Tampe, ${ }^{* \dagger}$ Valerie LeBleu, ${ }^{\dagger \dagger}$ Desiree Tampe, ${ }^{* \dagger}$ Elisabeth M. Zeisberg, ${ }^{\dagger \S \Phi}$ and Raghu Kalluri ${ }^{\dagger \dagger}$
}

\begin{abstract}
From the Departments of Nephrology and Rheumatology* and Cardiology and Pneumology, ${ }^{\S}$ Göttingen University Medical Center, Georg August University, Göttingen, Germany; the Division of Matrix Biology, ${ }^{\dagger}$ Department of Medicine, Beth Israel Deaconess Medical Center, Harvard Medical School, Boston, Massachusetts; the Department of Cancer Biology and the Metastasis Research Center, ${ }^{\ddagger}$ University of Texas MD Anderson Cancer Center, Houston, Texas; and the German Center for Cardiovascular Research (DZHK), "Göttingen, Germany
\end{abstract}

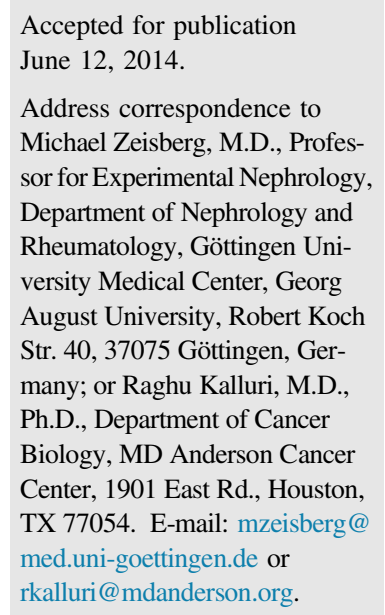

\begin{abstract}
Thrombospondin-1 (TSP1) is a multifunctional matricellular protein known to promote progression of chronic kidney disease. To gain insight into the underlying mechanisms through which TSP1 accelerates chronic kidney disease, we compared disease progression in Col4a3 knockout (KO) mice, which develop spontaneous kidney failure, with that of Col4a3;Tsp1 double-knockout (DK0) mice. Decline of excretory renal function was significantly delayed in the absence of TSP1. Although Col4a3;Tsp1 DK0 mice did progress toward end-stage renal failure, their kidneys exhibited distinct histopathological lesions, compared with creatinine levelmatched Col4a3 K0 mice. Although kidneys of both Col4a3 K0 and Col4a3;Tsp1 DK0 mice exhibited a widened tubulointerstitium, predominant lesions in Col4a3 K0 kidneys were collagen deposition and fibroblast accumulation, whereas in Col4a3;Tsp1 DKO kidney inflammation was predominant, with less collagen deposition. Altered disease progression correlated with impaired activation of transforming growth factor- $\beta 1$ (TGF- $\beta 1$ ) in vivo and in vitro in the absence of TSP1. In summary, our findings suggest that TSP1 contributes to progression of chronic kidney disease by catalyzing activation of latent TGF- $\beta 1$, resulting in promotion of a fibroproliferative response over an inflammatory response. Furthermore, the findings suggest that fibroproliferative and inflammatory lesions are independent entities, both of which contribute to decline of renal function. (Am J Pathol 2014, 184: 2687-2698; http://dx.doi.org/10.1016/j.ajpath.2014.06.014)
\end{abstract}

Progression of chronic kidney disease (CKD) toward endstage renal failure (ESRF) is a prominent problem in clinical nephrology. ${ }^{1}$ The incidence of CKD is rising, but effective therapies to halt progression of disease remain elusive. ${ }^{2}$ Progression of CKD results from a complex interplay of pathologies that involve all constituents of the kidney, which makes it difficult to single out targets for effective therapeutic strategies. $^{3}$

The extent of so-called tubulointerstitial fibrosis is often considered to be the rate-limiting step in progression of CKD. ${ }^{1}$ This idea is founded on histopathological analysis of large cohorts of kidney biopsies, which demonstrated that only tubulointerstitial fibrosis (which at the time was determined as the relative volume of the interstitium within a kidney biopsy section) correlates with and also predicts progression of CKD toward ESRF, irrespective of the underlying primary disease. ${ }^{4-7}$ Widening of the tubulointerstitium, which is referred to as tubulointerstitial fibrosis, is caused by a composite of extracellular matrix (ECM) accumulation, sterile inflammation, accumulation of activated fibroblasts, and rarefaction of microvessels. ${ }^{1}$ Although the relevance of each of these events to progression of fibrosis and CKD is hotly debated, this knowledge led to the concept that tubulointerstitial fibrosis is a common pathway of all chronic progressive kidney diseases and that effective antifibrotic therapies could

Supported by NIH grants R01DK55001-14, DK81576, CA125550, CA155370, CA151925, and CA163191 (R.K.); Metastasis Research Center; by German Research Foundation (DFG) grants ZE523/2-1 and ZE523/3-1 (M.Z.) and SFB1002/TPC01 (E.Z.); the Cancer Prevention and Research Institute of Texas (R.K.); and the seed funding research program of the Faculty of Medicine, Georg August University Göttingen (B.T., D.T.).

Disclosures: None declared. 
potentially halt progression of CKD irrespective of the underlying disease. However, such therapies are not yet available. ${ }^{1}$

Our aim was to gain insight into mechanisms that underlie the contribution of thrombospondin-1 (TSP1) to progression of CKD. TSP1 is the most-studied member of the thrombospondin family of matricellular proteins. ${ }^{8}$ Previous studies have demonstrated that pharmacological suppression or genetic depletion of TSP1 attenuates disease progression in animal models of CKD. ${ }^{9-13}$ TSP1 is a $450-\mathrm{kDa}$ trimeric ECM protein, which does not fulfill primarily structural roles in the matrix, but instead functions as an extracellular modulator of cell function. ${ }^{8,14}$ Most prominently, TSP1 is known to inhibit angiogenesis, inhibit inflammation, activate MMP-dependent ECM turnover, and facilitate fibroblast migration and activation, all of which are considered important contributors to progression of CKD. ${ }^{8,10}$ To delineate through which of its known biological activities TSP1 impacts progression of CKD, we compared progression of kidney disease of Col4a3 knockout (KO) mice (deficient in type IV collagen $\alpha 3$ chain) with that of Col4a3;Tsp1 double-knockout (DKO) mutant mice. ${ }^{15}$

Here, we demonstrate that decrease of excretory renal function is delayed if TSP1 is absent. Furthermore, tissue analysis of plasma creatinine level-matched kidneys of Col4a3 KO and of Col4a3;Tsp1 DKO revealed that in Col4a3 KO mice disease progression is predominantly associated with fibrosis, whereas inflammation is the predominant interstitial pathology in Col4a3;Tsp1 DKO mice. We provide evidence that this altered disease progression is due to impaired activation of latent transforming growth factor- $\beta 1$ (TGF- $\beta 1$ ) in the absence of TSP1. Our findings provide evidence that both fibroproliferative injury and inflammation can independently cause expansion of the interstitium, leading to decline of excretory renal function.

\section{Materials and Methods}

\section{Mice}

Col4a3 KO mice and Tsp1 KO mice, both on a C57BL/6 background, have been described previously. ${ }^{16-18}$ To establish double-mutant mice, Col4a3 KO mice and TspI $\mathrm{KO}$ mice were crossed, producing $\mathrm{Col}_{4 a 3^{+/-}} ; \mathrm{TspI}^{+/-}$mice in the $\mathrm{F}_{1}$ generation. Interbreeding of $\mathrm{Col}_{4 \mathrm{al}^{+/-}} ; \mathrm{Tsp}^{+/-}$ mice resulted in Col4a3 KO, Tsp1 KO, and Col4a3;Tspl DKO mice in the expected Mendelian ratio. Mice were genotyped by PCR using established primers and protocols. ${ }^{16,17}$ The TSP1-deficient mice were provided by Dr. Jack Lawler (Beth Israel Deaconess Medical Center, Boston, MA). All procedures were performed with the approval of the Institutional Animal Care and Use Committee of the Beth Israel Deaconess Medical Center.

\section{Histological Analysis}

Formalin-fixed, paraffin-embedded kidneys were sectioned at $3 \mu \mathrm{m}$ and stained with hematoxylin and eosin (H\&E),
Masson's trichrome stain (MTS), or periodic acid-Schiff's stain (PAS) at the Beth Israel Deaconess Medical Center (Boston, MA) Histopathology Core facility. The relative interstitial volume was evaluated by morphometric analysis using a counting grid, as described previously, ${ }^{19}$ and 100 points per visual field $(\times 60$ magnification) were identified as tubule or interstitium. Results were summarized as interstitium among all scored areas (relative percentage of interstitial area). The fibrotic area was assessed using image processing software (Image-Pro Plus version 6.2; Media Cybernetics, Rockville, MD) as described previously. ${ }^{20}$ In brief, the blue (fibrotic) and nonblue areas were measured in photomicrographs at $\times 20$ magnification. Glomerulosclerosis was similarly assessed with Image-Pro Plus software. Glomerulosclerosis was quantified as the PAS-positive area per glomerulus $(\times 60$ magnification). To assess cellularity, we counted all visible nuclei per visual field $(\times 60$ magnification) of H\&E-stained sections. To assess the tubular atrophy index, tubules were evaluated for widened lumen and thickened basement membranes and the percentage of atrophic tubules was estimated. ${ }^{19}$

\section{Staining Procedures}

Immunolabeling was performed on formalin-fixed, paraffinembedded kidney sections. Sections were deparaffinized and microwaved for 15 minutes in antigen unmasking solution (Vector Laboratories, Burlingame, CA). For immunohistochemistry, sections were blocked with avidin-biotin and $10 \%$ horse serum (both from Vector Laboratories) before incubation with the respective primary antibodies: $\alpha \mathrm{SMA}$ and GPR44 (LifeSpan BioSciences); CD45 (BD Biosciences, San Jose, CA); CCR5, IL-13, ROR- $\gamma$, and FOXP3 (Abcam, Cambridge, MA); and TSP1 and p-SMAD2/3 (Santa Cruz Biotechnology, Dallas, TX). Immunolabeling was visualized using a Vectastain alkaline phosphatase kit with alkaline phosphatase substrate kit I (Vector Laboratories) according to the manufacturer's recommendations. For immunofluorescence staining, kidney sections were incubated with primary antibodies to CD31 and collagen IV. Collagen IV antibodies were raised against the noncollagenous domain of type IV collagen and therefore detected all type IV collagen isoforms, including collagen IV $\alpha 1$ and/or $\alpha 2$ chains, which are abundantly present in the Col4a3 KO and Col4a3;Tsp1 KO mice. Nuclei were counterstained with DAPI. Images were captured with confocal microscopy.

\section{Preparation of Primary Renal Fibroblasts}

Primary renal fibroblasts were isolated from kidneys of C57BL/6 wild-type mice and of Tspl KO mice as described previously ${ }^{21}$ with minor modifications. Kidney cortex from 6-month-old mice was minced and digested in $100 \mathrm{U} / \mathrm{mL}$ type II collagenase (Worthington Biochemical, Lakewood, $\mathrm{NJ}$ ) for 30 minutes at $37^{\circ} \mathrm{C}$. The tissue pieces (approximately 
A
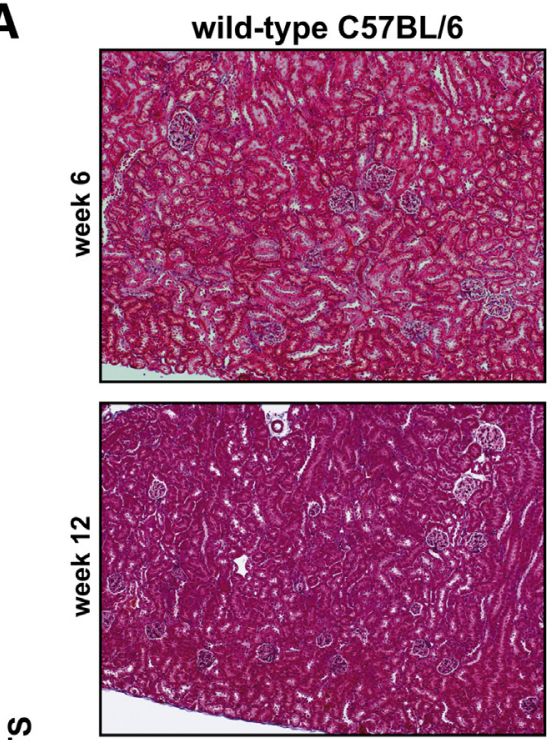

$\stackrel{n}{\Sigma}$
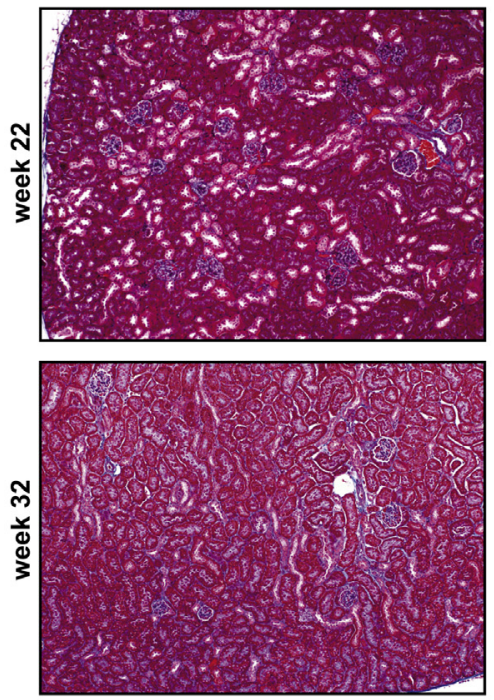

Col4a3 KO
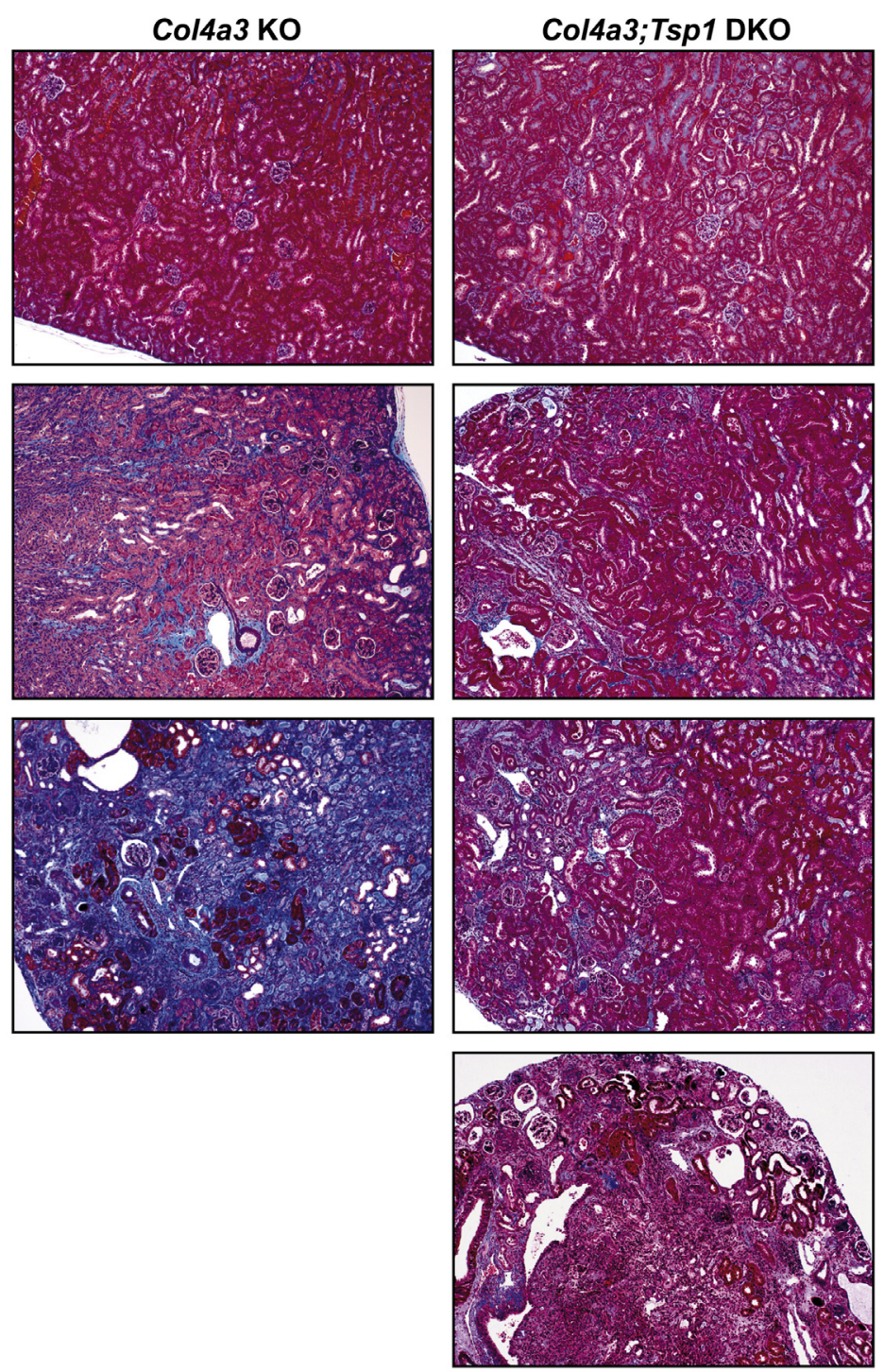

Figure 1 Progression of CKD in Col4a3 K0 and Col4a3;Tsp1 DK0 mice. A: Representative photomicrographs of MTS-stained kidney sections of wild-type C57BL/ 6 control, Col4a3 K0, and Col4a3;Tsp1 DK0 mice at 6, 12, 22, and 32 weeks. Col4a3 K0 mice did not live to 32 weeks of age. B: Serum creatinine levels of wild-type C57BL/6, Tsp1 K0, Col4a3 K0 mice, and Col4a3;Tsp1 DKO mice over time. Data are expressed as means \pm SEM. Original magnification, $\times 10$. CKD, chronic kidney disease; DK0, double knock-out; ESRF, end-stage renal failure; K0, knock-out; MTS, Masson's trichrome stain.
B

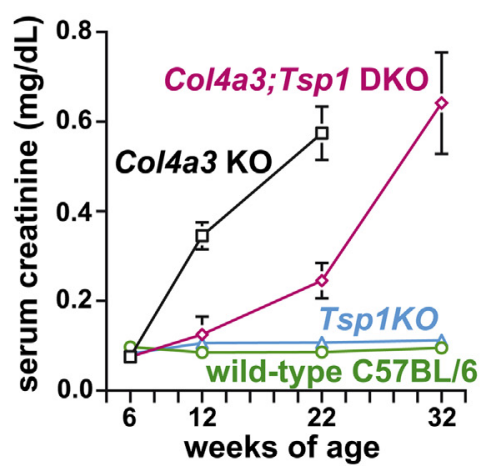

$5 \mathrm{~mm}^{3}$ ) were washed to remove the collagenase and then were plated into T25 tissue-culture flasks in Dulbecco's modified Eagle's medium containing 10\% fetal bovine serum and antibiotics $(100 \mathrm{U} / \mathrm{mL}$ penicillin $\mathrm{G}, 100 \mathrm{~g} / \mathrm{mL}$ streptomycin, and $0.25 \mu \mathrm{g} / \mathrm{mL}$ amphotericin B).

\section{Proliferation Assay}

Fibroblast proliferation in response to active and latent TGF- $\beta 1$ was assayed as described previously, ${ }^{21}$ with minor modifications. Primary wild-type and Tspl KO fibroblasts 
A

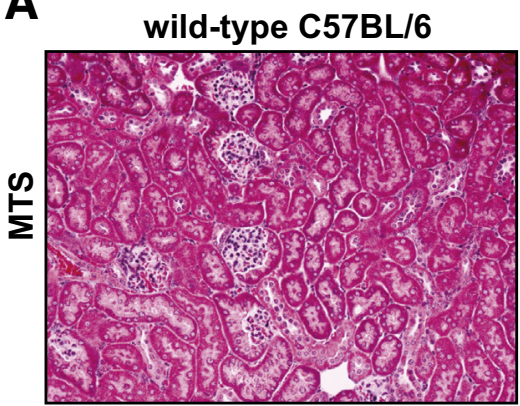

B

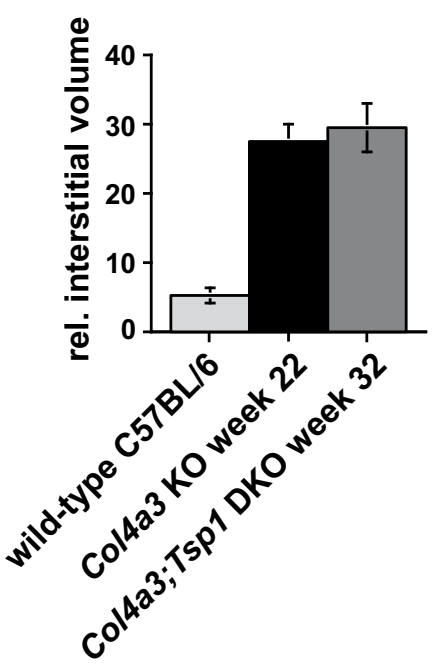

Col4a3 KO week 22

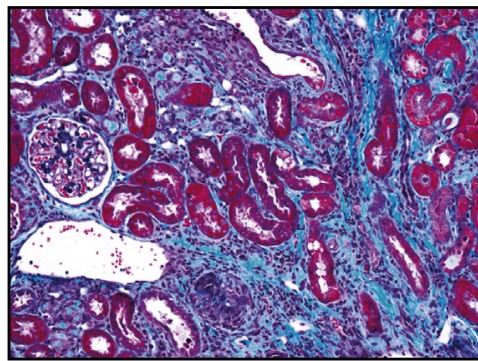

C

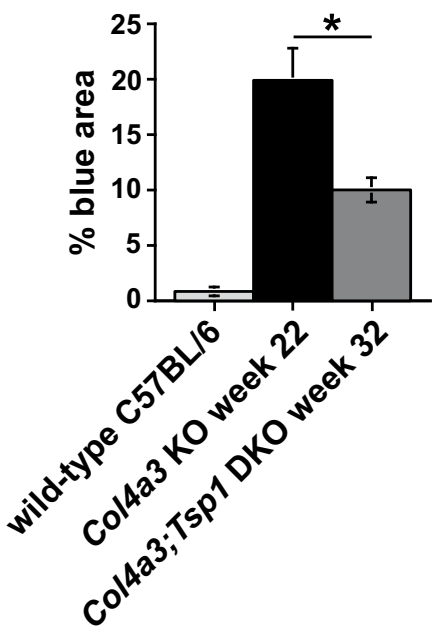

Col4a3;Tsp1 DKO week 32

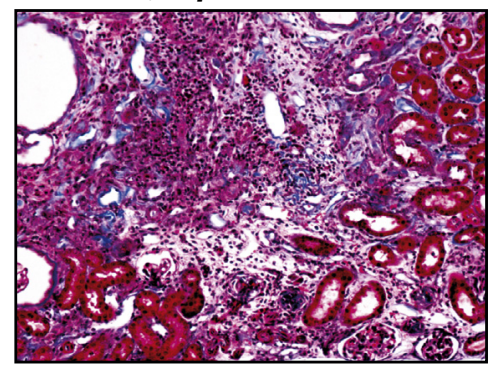

D

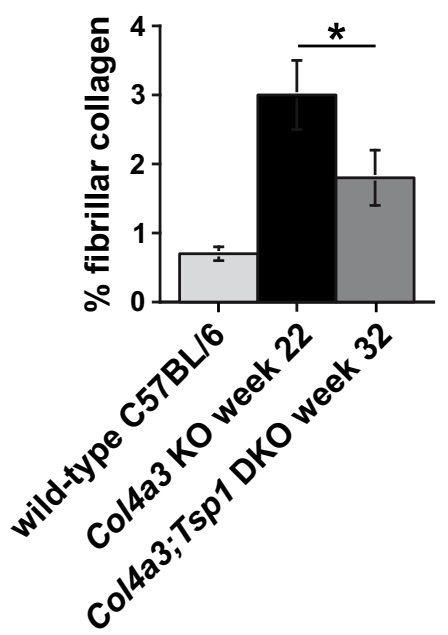

Figure 2 Histopathology of fibrosis. A: Representative photomicrographs of MTS-stained kidney sections of wild-type control (32 weeks) and creatininematched Col4a3 KO and Col4a3;Tsp1 DKO mice. B: The degree of tubulointerstitial fibrosis (determined by relative interstitial volume) did not differ significantly between kidneys of Col4a3 K0 and Col4a3;Tsp1 DK0 mice. C: In MTS-stained kidney sections, the degree of collagen deposition (blue) was significantly higher in Col4a3 K0 mice than in Col4a3;Tsp1 DK0 mice. D: Fibrillar collagen content (determined as percent of total kidney protein) was higher in Col4a3 K0 kidneys than in creatinine-matched Col4a3;Tsp1 DK0 kidneys. Data are expressed as means \pm SEM. ${ }^{*} P<0.05$. Original magnification, $\times 20$.

were seeded into 96-well plates (4000 cells per well). On 24 hours of incubation in serum-free Dulbecco's modified Eagle's medium, the medium was exchanged for medium containing $5 \mathrm{ng} / \mathrm{mL}$ active or latent TGF- $\beta 1$ (R\&D Systems). Cell proliferation was measured using a colorimetric cell proliferation assay (WST-1-based, Roche) according to the manufacturer's recommendations.

\section{Quantification of Fibrillar Collagen Content}

Fibrillar collagen was quantified in kidney protein lysates using a QuickZyme (Leiden, The Netherlands) fibrillar collagen assay kit according to the manufacturer's recommendations. In brief, kidney tissues were homogenized in $0.5 \mathrm{~mol} / \mathrm{L}$ acetic acid-pepsin. Total protein was
A

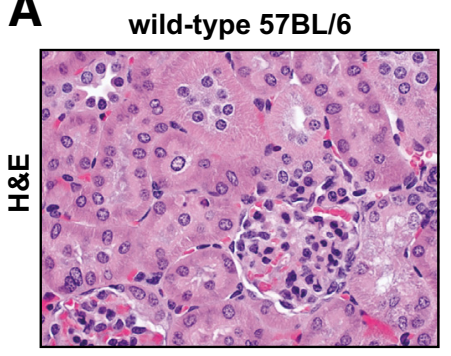

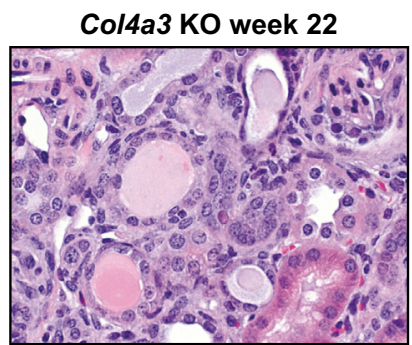

Col4a3;Tsp1 DKO week 32

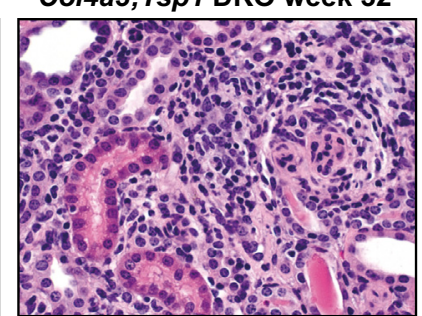

B

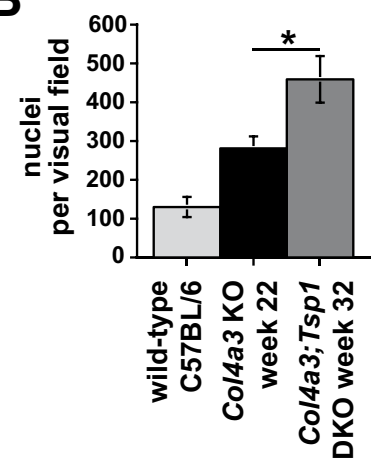

Figure 3 Interstitial cellularity. A: Representative photomicrographs of H\&E-stained kidney sections of wild-type control and creatinine-matched Col4a3 $\mathrm{KO}$ and Col4a3;Tsp1 DKO mice. B: The number of nuclei per visual field was significantly lower in kidneys of Col4a3 K0 mice, compared with Col4a3;Tsp1 DKO mice. Data are expressed as means $\pm \mathrm{SEM} .{ }^{*} P<0.05$. Original magnification, $\times 60$. H\&E, hematoxylin and eosin. 
A
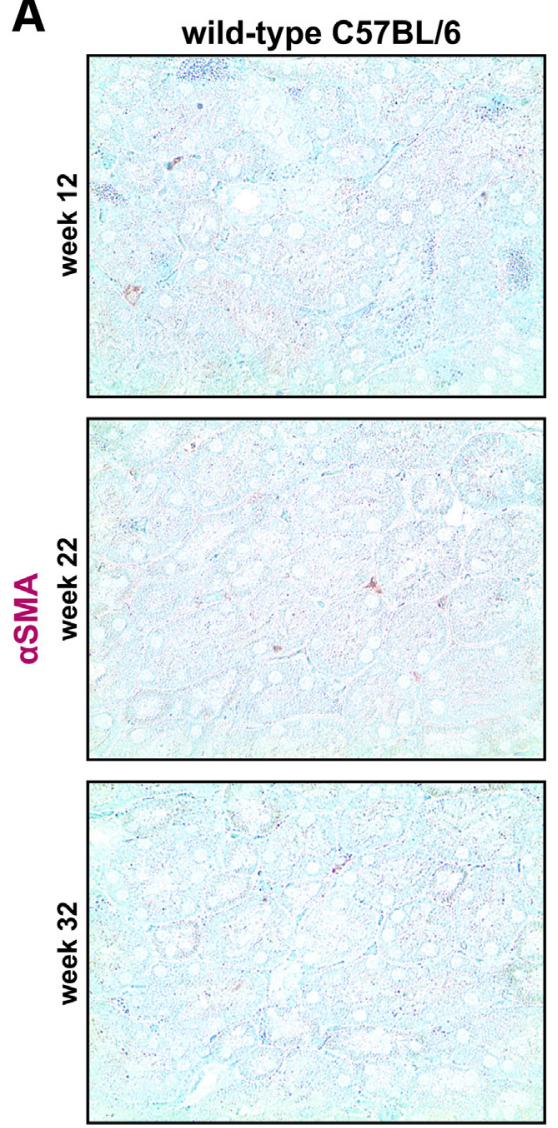

Col4a3 KO
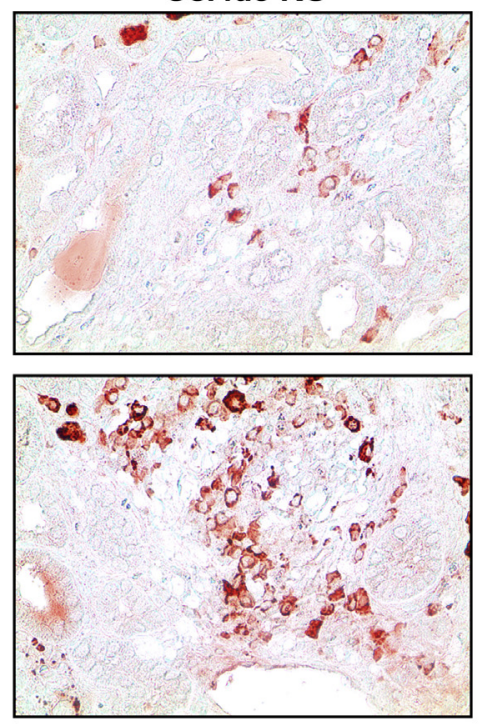

Col4a3;Tsp1 DKO
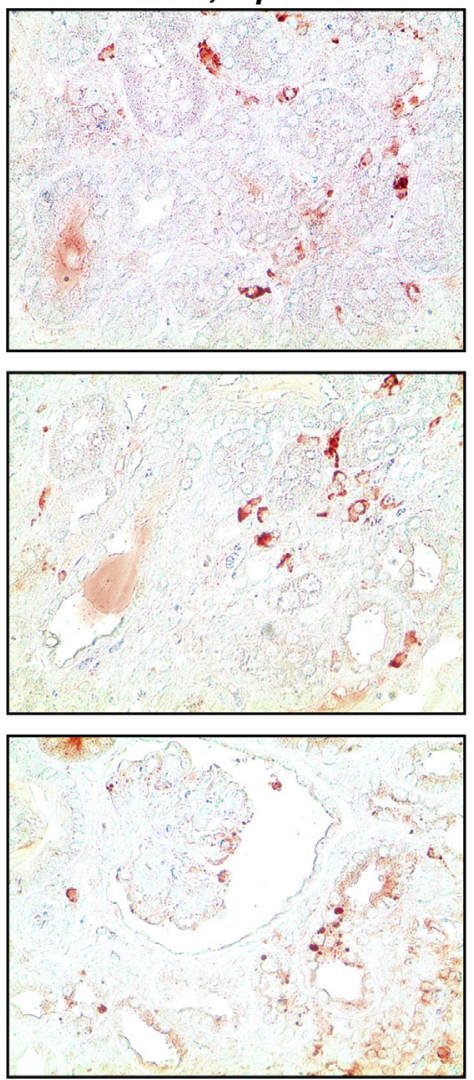

B

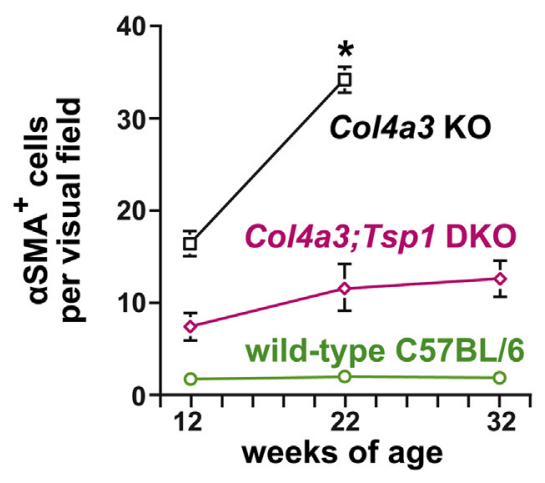

Figure 4 Fibroblast accumulation during progression of CKD in Col4a3 KO and Col4a3;Tsp1 DK0 mice. A: Representative photomicrographs of formalinfixed, paraffin-embedded kidney sections of wild-type control, Col4a3 K0, and Col4a3;Tsp1 DK0 mice at 12, 22, and 32 weeks of age. Sections were immunolabeled with antibodies against $\alpha$ SMA (red) and counterstained with methylene green. B: Fibroblast numbers were determined as $\alpha \mathrm{SMA}^{+}$cells per visual field. The fibroproliferative response over time was more robust in Col4a3 K0 mice than in Col4a3;Tsp1 DKO mice and differences reached statistical significance at 22 weeks of age. Data are expressed as means $\pm \mathrm{SEM}$. ${ }^{\star} P<0.05$. Original magnification, $\times 20$. aSMA, $\alpha$-smooth muscle actin. quantified, and $100 \mu \mathrm{g}$ per well of each sample was analyzed in the kit's 96-well plates. A standard curve using the kit's collagen standard was generated, and a best-fit linearized curve was generated to determine collagen content per well.

\section{RNA Isolation and Quantitative Real-Time PCR}

Total RNA was isolated using Trizol reagent (Life Technologies, Carlsbad, CA) according to the manufacturer's recommendation. Specific primers to collagen III $\alpha 1$ were designed using the Primer Express 1.5 program. ${ }^{21}$ TaqMan Gene Expression Assay (Life Technologies) were used for quantitative real-time PCR performed with an ABI 7000 sequence detection system (Life Technologies), and measurements were standardized to 18S rRNA (Life Technologies) using standard calculation methods as described previously. ${ }^{22}$

\section{Statistical Analysis}

Statistical analysis was performed using the Kruskal-Wallis method followed by Dunn's test. If equal variance test failed, then $U$-test was used. All statistical analysis was performed using SigmaStat 2.0 software (Systat, San Jose, CA). 
A
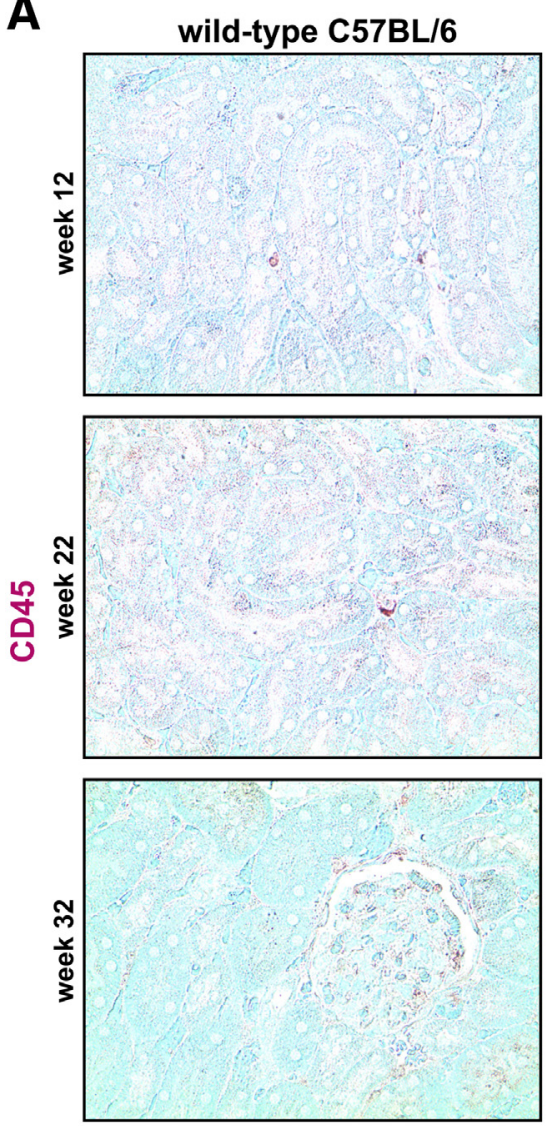

Col4a3 KO
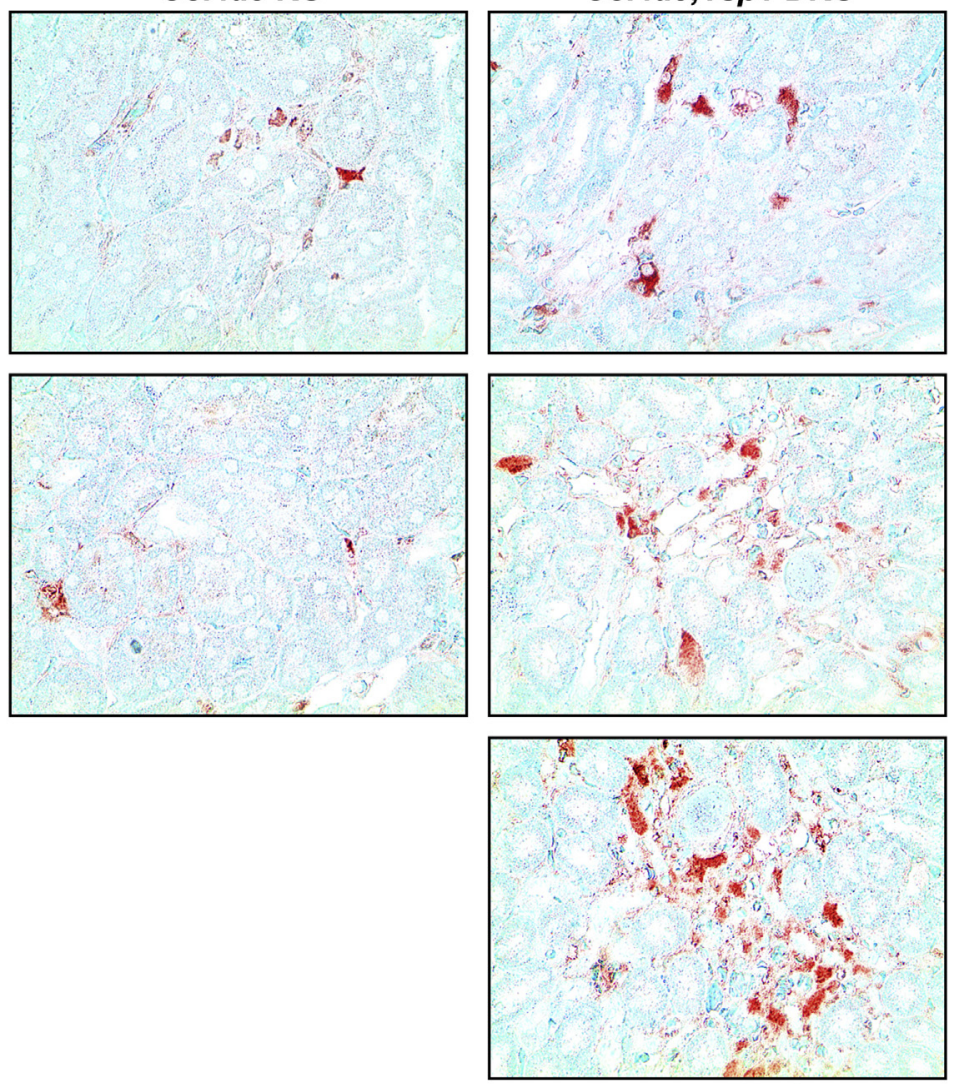

B

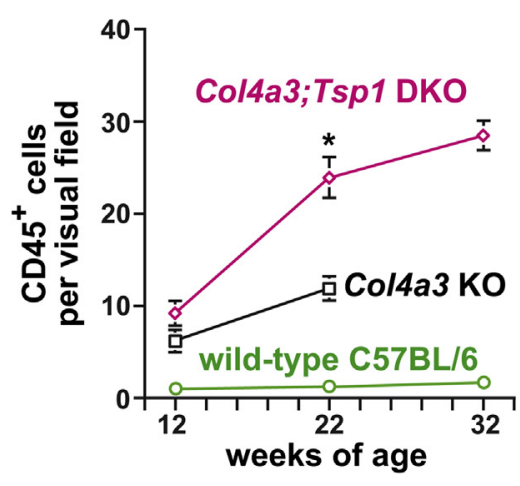

Figure 5 Intrarenal accumulation of inflammatory cells in the progression of CKD in Col4a3 K0 and Col4a3;Tsp1 DK0 mice. A: Representative photomicrographs of formalin-fixed, paraffin-embedded kidney sections of wild-type control, Col4a3 K0, and Col4a3;Tsp1 DK0 mice at 12, 22, and 32 weeks of age. Sections were immunolabeled with antibodies against CD45 (red) and counterstained with methylene green. B: Inflammation was determined as numbers of $\mathrm{CD}_{4} 5^{+}$inflammatory cells per visual field. The inflammatory response over time was more robust in Col4a3;Tsp1 DK0 mice than in Col4a3 $\mathrm{KO}$ mice and reached statistical significance at 22 weeks of age. Data are expressed as means \pm SEM. ${ }^{*} P<0.05$. Original magnification, $\times 20$.

\section{Results}

Progression of Renal Fibrogenesis Is Delayed in Col4a3;Tsp1 DK0 Mice

We compared progression of kidney disease of Col4a $3 \mathrm{KO}$ with that of Col4a3;Tsp1 DKO mutant mice. ${ }^{15}$ Col4a3 KO mice were originally generated as a mouse model for Alport syndrome; on a C57BL/6 genetic background, these mice exhibit robust and reproducible progression of spontaneous kidney disease toward ESRF due to a defined genetic defect. ${ }^{23}$ In Col4a 3 KO mice, absence of the $\alpha 3$ chain of type IV collagen leads to glomerular basement membrane

splitting and proteinuria starting at 6 weeks of age. Tubulointerstitial lesions (including ECM accumulation, fibroblast accumulation, sterile inflammation, and microvascular rarefaction) can be detected starting at 12 weeks of age. ${ }^{23}$ At the 22 weeks of age, kidneys of Col4a3 KO mice are severely fibrotic, and mice die of ESRF at the age of 24 to 26 weeks. $^{23}$ Col4a3 KO mice on a C57BL/6 background allow for analysis of all aspects of chronic progressive kidney disease. ${ }^{23}$

We sacrificed predetermined cohorts of mice (Col4a3 KO, Col4a3;Tsp1 DKO, and C57BL/6 wild-type control mice) at $6,12,22$, and 32 weeks of age. This study design was founded on our previous studies, which demonstrated that 

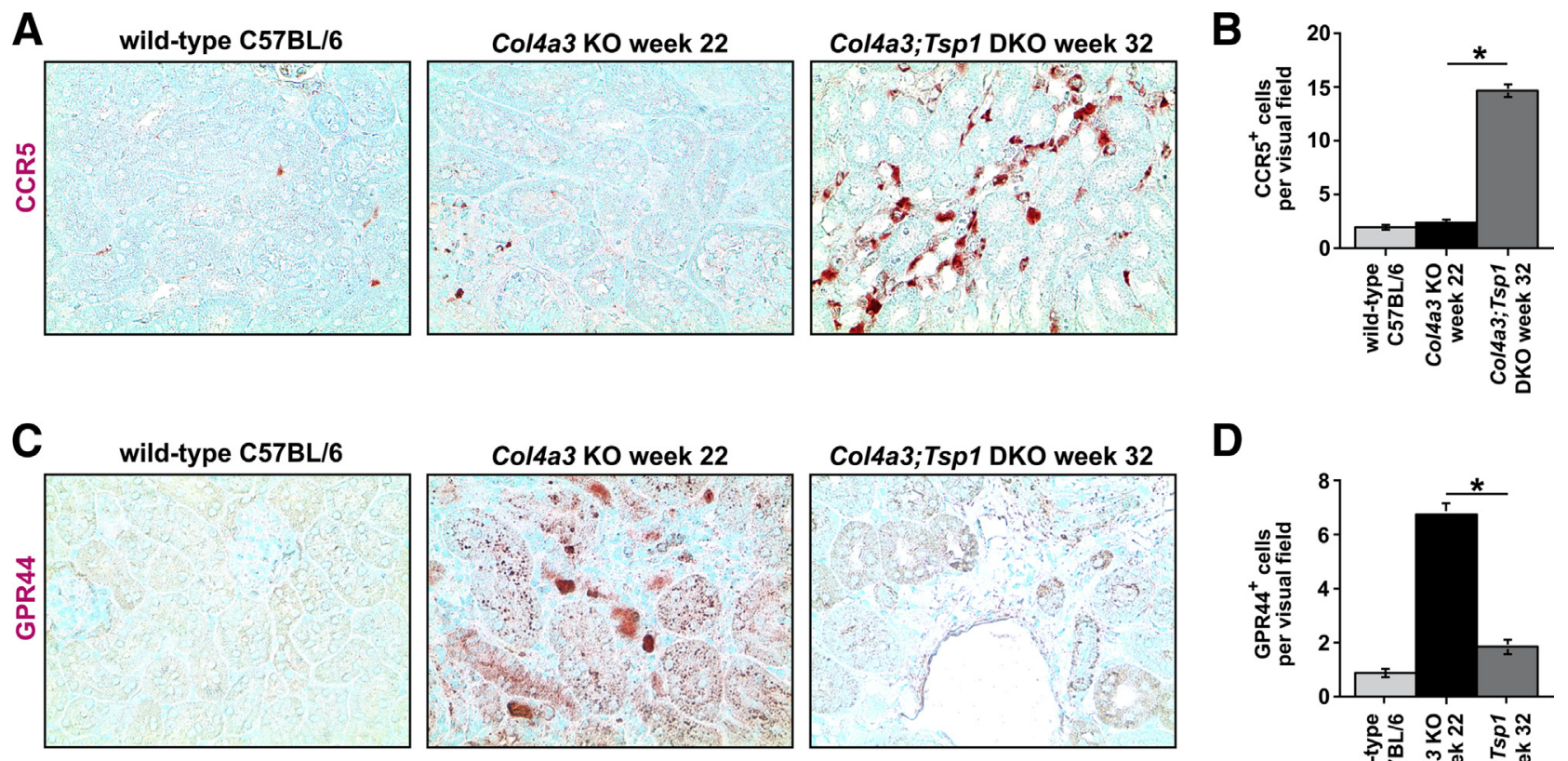

D

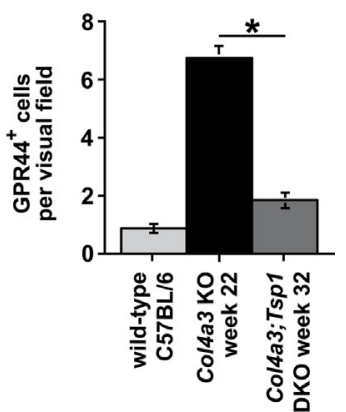

Figure 6 Interstitial accumulation of Th1 and Th2 cells. A and C: Representative microphotographs of formalin-fixed, paraffin-embedded kidney sections of wild-type control (32 weeks) and creatinine-matched Col4a3 KO and Col4a3;Tsp1 DKO mice. Sections were labeled with antibodies against C-C chemokine receptor type 5 (CCR5, a marker of Th1 cells) (A) or with G-protein coupled receptor 44 (GPR44, a marker of Th2 cells) (C) and counterstained with methylene green. B and D: Numbers of CCR5 ${ }^{+}$T cells (B) and GPR44 ${ }^{+}$T cells (D) per visual field. Col4a3 K0 mice exhibited interstitial accumulation of GPR44 ${ }^{+}$Th2 cells; in creatinine-matched Col4a3;Tsp1 DK0 mice, TSP1 deficiency was associated with a Th1 switch and accumulation of CCR5 ${ }^{+}$Th1 cells. Data are expressed as means \pm SEM. ${ }^{*} P<0.05$. Original magnification, $\times 40$.

Col4a3;Tsp1 DKO mice survive to only 36 weeks of age, whereas Tsp1 KO mice with normal kidney function have a normal life span. ${ }^{15}$ MTS-stained sections of kidney tissue from Col4a3 KO mice indicated absence of interstitial fibrosis at the age of 6 weeks, but these mice had developed fibrosis by 12 weeks of age, with severe fibrosis at 22 weeks, confirming previous studies (Figure $1 \mathrm{~A}$ ). ${ }^{24}$ The progression of chronic kidney injury observed in MTS-stained sections correlated with progressive impairment of excretory renal function, as determined by plasma creatinine levels (Figure 1B). Compared with Col4a3 KO mice, decline of excretory renal function was delayed in Col4a3;Tspl DKO mice; plasma creatinine levels after 32 weeks were similar to those observed in the Col4a3 KO mice after 22 weeks (Figure 1B). Such delayed disease progression correlated with histopathological lesions noted in MTS-stained kidneys.

\section{Distinct Histopathologies in Fibrotic Kidneys of Col4a3 KO and Col4a3;Tsp1 DKO Mice}

MTS-stained sections of end-stage diseased kidneys of Col4a3 KO mice (at 22 weeks) and of Col4a3;Tsp1 DKO mice (at 32 weeks) had markedly different pathologies (Figure 1), despite similar plasma creatinine levels. We therefore decided to systematically compare kidneys of creatinine-matched cohorts, as opposed to age-matched cohorts, to gain further insight into the role of TSP1 in renal fibrogenesis. We chose this approach of comparing kidneys with similar impairment of function over that of comparing age-matched kidneys of each group because we found that for kidneys at 22 weeks all parameters appeared to be improved in the Col4a3;Tspl DKO mice, providing little insight into why disease progression was delayed.

We first assessed the relative interstitial volume (ie, the cortical area not covered by glomeruli or tubules) in MTSstained kidney sections of wild-type C57BL/6 (32 weeks) and of end-stage diseased Col4a3 KO and Col4a3;Tspl DKO mice (Figure 2). The interstitial volume was significantly increased in both Col4a3 KO and Col4a3;Tspl DKO kidneys, compared with the wild type, with no significant difference between the two mutant mouse groups (Figure 2). However, when we digitally quantified the relative blue area (reflective of collagen deposition) in MTS-stained kidney sections, we found that the relative blue area was significantly smaller in the Col4a3;Tsp1 DKO kidneys than in the creatinine-matched Col4a3 KO kidneys (Figure 2C). To confirm that reduced blue staining in the Col4a3;Tspl DKO kidneys is reflective of decreased collagen deposition, we analyzed fibrillar collagen content (per $100 \mu \mathrm{g}$ total protein lysate) of kidney tissue. Collagen content in functionally impaired Col4a3;Tsp1 DKO kidneys was significantly reduced, compared with creatinine-matched Col4a3 KO kidneys (Figure 2D). In creatinine-matched kidneys, the Col4a3;Tsp1 DKO kidneys contained less collagen than 

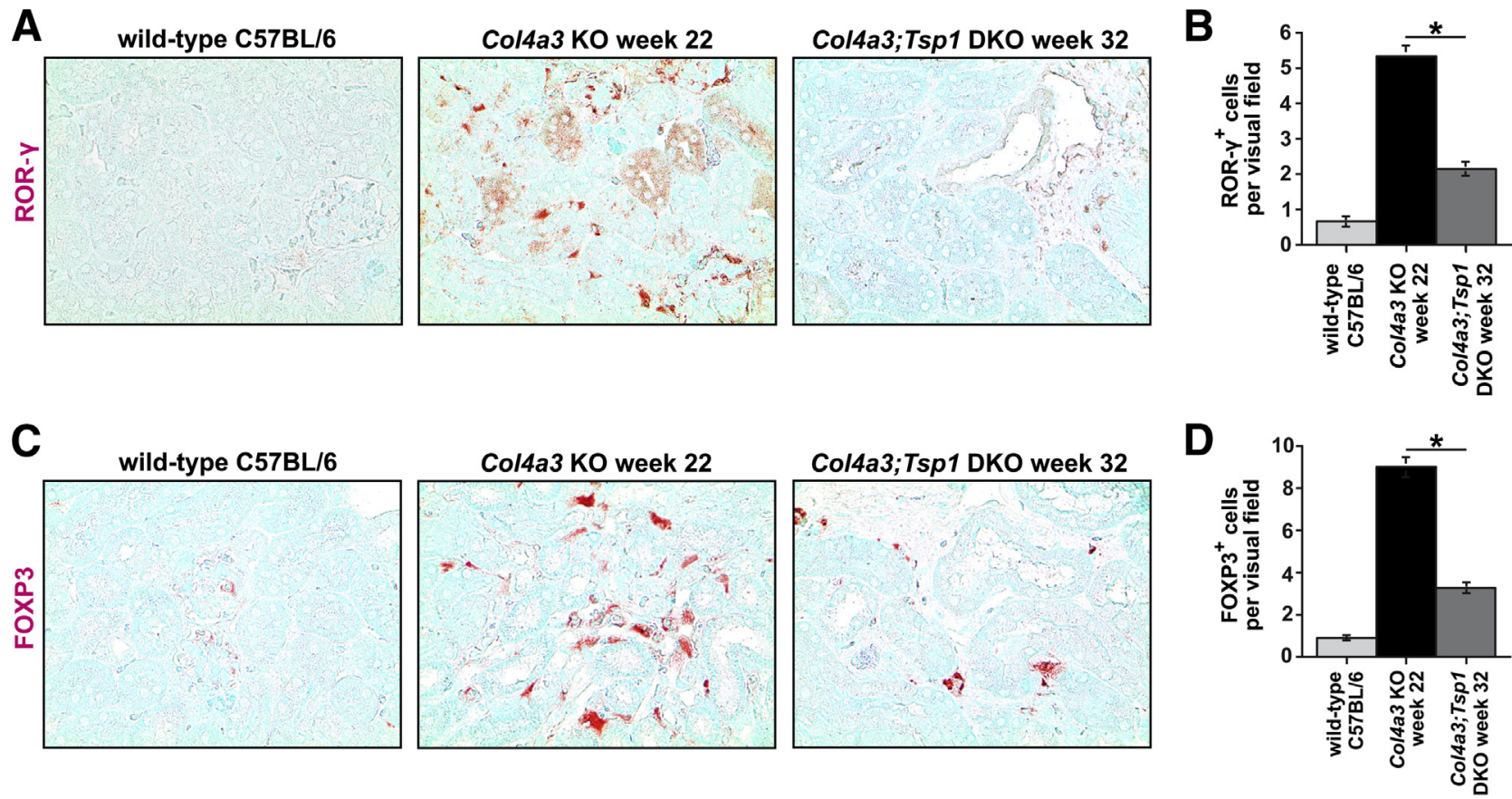

Figure 7 Accumulation of Th17 and T-regulatory cells. A and C: Representative microphotographs of formalin-fixed, paraffin-embedded kidney sections of wildtype control (32 weeks) and creatinine-matched Col4a3 K0 and Col4a3;Tsp1 DKO mice. Sections were labeled with antibodies against RAR-related orphan receptor- $\gamma$ (ROR- $\gamma$, a marker of Th17 cells) (A) or forkhead box protein P3 (FOXP3, a marker of T-regulatory cells) (C) and counterstained with methylene green. B and D: Numbers of ROR- $\gamma^{+}$(B) and FOXP3 ${ }^{+}$(D) T cells per visual field. TSP1 deficiency in creatinine-matched Col4a3;Tsp1 DKO mice was associated with loss, relative to Col4a3 K0 mice, of Th17 cells $\left(\mathrm{ROR}-\gamma^{+}\right)$and T-regulatory cells $\left(\mathrm{FOXP} 3^{+}\right)$. Data are expressed as means $\pm \mathrm{SEM} .{ }^{*} P<0.05$. Original magnification, $\times 40$.

Col4a3 KO kidneys, despite similar expansion of the interstitial space.

\section{Identity of Interstitial Cells in Fibrotic Kidneys of Col4a3 K0 and Col4a3;Tsp1 DKO Mice}

We next compared interstitial cellularity (determined in H\&Estained sections as nuclei per visual field) between kidneys of creatinine-matched Col4a3 KO and Col4a3;Tsp1 DKO mice.
Cellularity was significantly increased in Col4a3;Tspl DKO kidneys, compared with creatinine-matched Col4a3 KO kidneys (Figure 3). To gain insight into the identity of the interstitial cells, we performed immunolabeling experiments using antibodies to the fibroblast marker $\alpha$-smooth muscle actin $(\alpha \mathrm{SMA})$ in which bone marrow-derived inflammatory cells were immunolabeled with CD45. In kidneys of 22-week-old Col4a $3 \mathrm{KO}$ mice, the number of $\alpha \mathrm{SMA}^{+}$fibroblasts per visual field was substantially increased, compared with wild-type
A

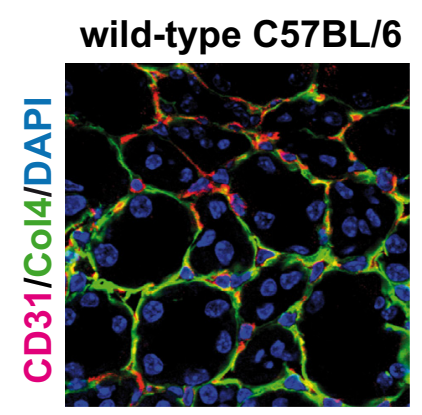

Col4a3 KO week 22

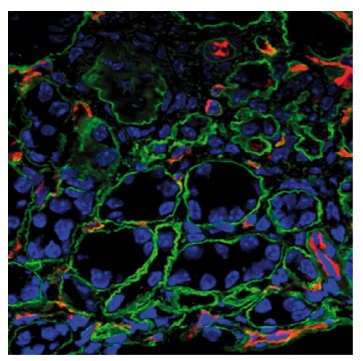

\section{Col4a3;Tsp1 DKO} week 32

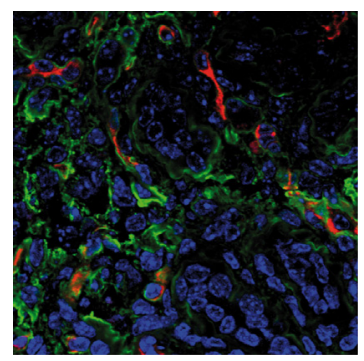

B

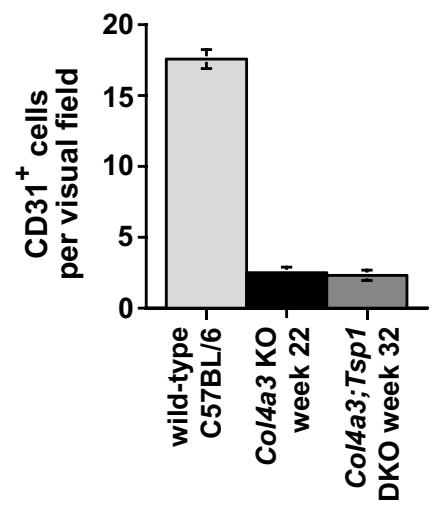

Figure 8 A: Representative photomicrographs of kidney sections of wild-type control (32 weeks) and creatinine-matched Col4a3 K0 and Col4a3;Tsp1 DK0 mice. Sections were immunolabeled with CD31 (red), a marker of vasculature; the basal membrane was stained with an antibody to collagen IV (green) that recognizes collagen IV $\alpha 1 / 2$ chains and thereby also detects collagen IV in Col4a3 K0 and Col4a3;Tsp1 DK0 mice. Nuclei were counterstained with DAPI. B: Numbers of microvascular intersections per visual field. TSP1 deficiency in Col4a3;Tsp1 DK0 mice had no additional effect on microvascular density, compared with Col4a3 K0 mice. Data are expressed as means \pm SEM. Original magnification, $\times 60$. Col4, collagen IV. 

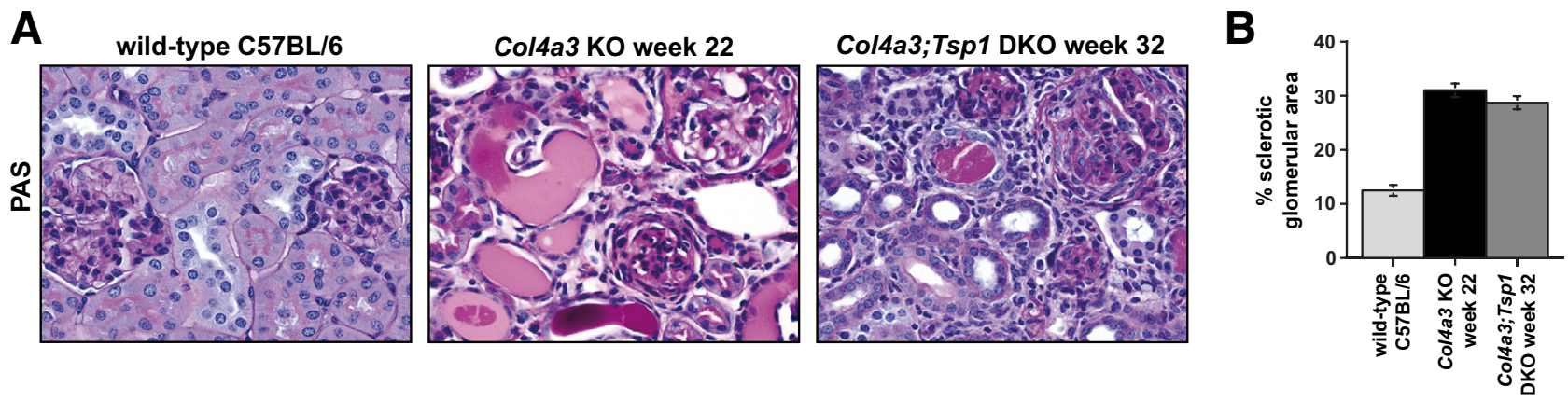

Figure 9 A: Representative photomicrographs of PAS-stained kidney sections of wild-type control (32 weeks) and creatinine-matched Col4a3 K0 and Col4a3;Tsp1 DK0 mice. B: Relative sclerotic glomerular area (PAS-positive area per glomerulus) did not differ significantly between kidneys of Col4a3 K0 mice and Col4a3;Tsp1 DKO mice. Data are expressed as means \pm SEM. Original magnification, $\times 60$. PAS, periodic acid-Schiff.

C57BL/6 control kidneys (Figure 4). The kidneys of 32-weekold creatinine-matched Col4a3;Tspl DKO mice exhibited significantly less $\alpha \mathrm{SMA}^{+}$interstitial fibroblast accumulation, compared with creatinine-matched Col4a3 KO kidneys (Figure 4). By contrast, CD45 ${ }^{+}$inflammatory cells were significantly increased in kidneys of 32-week-old Col4a3;Tsp1 DKO mice, compared with kidneys of creatinine-matched 22-week-old Col4a3 KO mice (Figure 5).

To further explore the contribution of different T-cell populations to the increase in inflammatory cells observed in Col4a3;Tsp1 DKO mice, we performed immunolabeling using antibodies to C-C chemokine receptor 5 (CCR5, a marker of Th1 cells), G-protein coupled receptor 44 (GPR44, a marker of
Th2 cells), RAR-related orphan receptor- $\gamma$ (ROR- $\gamma$, a marker of Th17 cells), or forkhead box protein P3 (FOXP3, a marker of T-regulatory cells). Fibroproliferative response in Col4a $3 \mathrm{KO}$ mice was associated with accumulation of GPR $44^{+} \mathrm{Th} 2$ cells (Figure 6, A and B); in creatinine-matched Col4a3;Tsp1 DKO mice, TSP1 deficiency caused a Th1 switch with accumulation of CCR5 ${ }^{+}$Th1 cells (Figure 6, C and D). This is consistent with recent findings describing a role of Th2 accumulation in progression of renal fibrogenesis and suggesting that inflammation not only changes quantitatively when TSP1 is absent, but also qualitatively, with a switch from a Th2 response to a Th1 response. ${ }^{25-28}$ Furthermore, TSP1 deficiency in Col4a3;Tsp 1 DKO mice was associated with loss of ROR- $\gamma^{+}$Th17 and
A

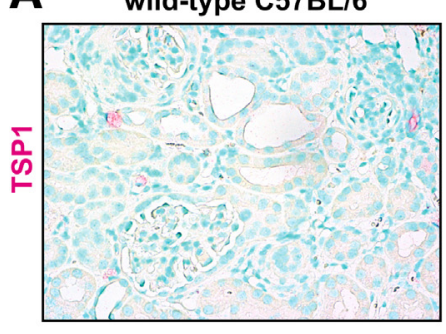

C

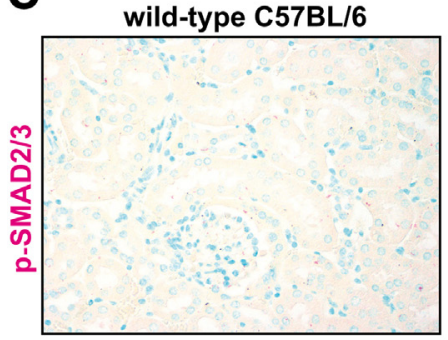

Col4a3 KO week 22

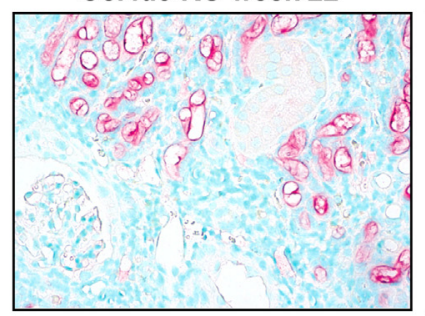

Col4a3 KO week 22

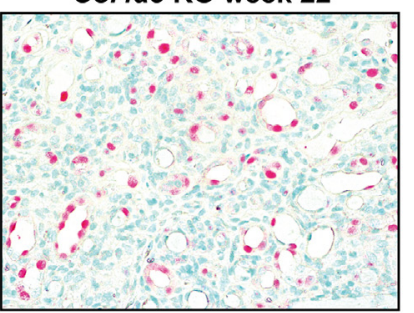

Col4a3;Tsp1 DKO week 32

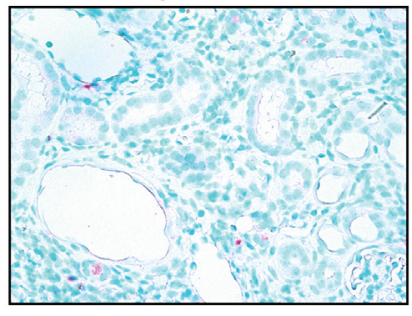

Col4a3;Tsp1 DKO week 32

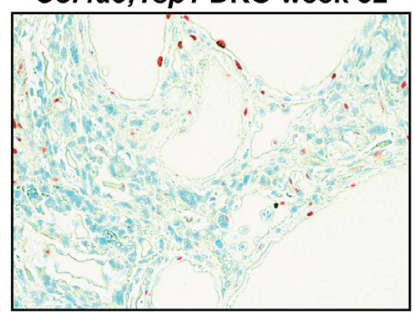

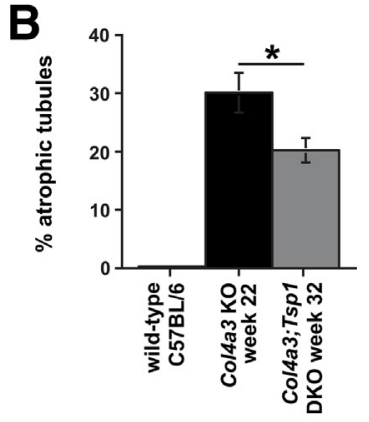

D

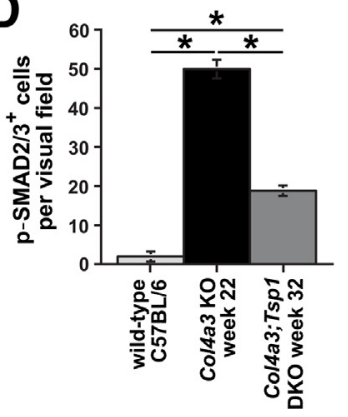

Figure 10 TSP1 deficiency attenuates TGF- $\beta 1$ signaling. A and C: Representative photomicrographs of formalin-fixed, paraffin-embedded kidney sections of wild-type control (32 weeks) and creatinine-matched Col4a3 K0 and Col4a3;Tsp1 DKO mice. A: Sections were labeled with antibodies to TSP1 (red) and counterstained with methylene green. In the fibrotic Col4a3 KO kidneys, TSP1 was localized predominantly in tubular epithelial cells, blood vessels, and interstitial cells. B: The percentage of atrophic tubules was significantly greater in kidneys of Col4a3 K0 mice than in Col4a3;Tsp1 DKO mice. C: Sections were labeled with antibodies to phosphorylated SMADs 2 and 3 (p-SMAD2/3), indicative of active signaling in response to TGF- $\beta 1$ (red) and counterstained with methylene green. D: Numbers of p-SMAD2 $/ 3^{+}$nuclei per visual field. SMAD2/3 signaling was significantly enhanced in tubular and interstitial compartments of kidneys of Col $4 a 3 \mathrm{KO}$ mice, compared with creatinine-matched Col4a3;Tsp1 DK0 kidneys. Data are expressed as means \pm SEM. ${ }^{*} P<0.05$. Original magnification, $\times 40$. 
$\mathrm{FOXP}^{+}$T-regulatory cells (Figure 7), supporting previous reports of a role of TSP1 in differentiation of Th17 and Tregulatory cells involved in regulating inflammatory response in the kidney. ${ }^{29-33}$ Because both TSP1 and the NC1 domain of the $\alpha 3$ chain of type IV collagen are inhibitors of tumor angiogenesis, and because rarefaction of microvessels is a consequence of tubulointerstitial fibrosis, ${ }^{1,15}$ we next compared microvascular density in kidneys of creatininematched Col4a3 KO and Col4a3;Tsp1 DKO mice. Analysis of CD31 immunostaining revealed that microvascular density was reduced in both Col4a3 $\mathrm{KO}$ and Col4a3;Tsp1 DKO mice, but did not differ in creatinine-matched kidneys (Figure 8).

In summary, inflammation (due to accumulation of Th1 cells and loss of Th17 and T-regulatory cells) was more robust in Col4a3;Tspl DKO kidneys, compared with creatinine-matched Col4a3 KO mice, but fibroblast accumulation was reduced.

\section{Glomerular Sclerosis and Tubular Atrophy in Fibrotic Kidneys of Col4a3 KO and Col4a3;Tsp1 DKO Mice}

In PAS-stained kidney sections, we digitally quantified deposition of PAS-positive material within glomeruli as an indicator of glomerulosclerosis. We found that glomerulosclerosis did not differ between Col4a3;Tsp1 DKO mice and creatininematched Col4a3 KO mice (Figure 9), suggesting that delayed fibrogenesis in Col4a3;Tsp1 DKO mice is not due to attenuated glomerular injury. This observation correlated with absence of glomerular TSP1 immunolabeling, even in sclerotic glomeruli of Col4a3 KO mice (Figure 10A), suggesting that TSP1 is not involved in progression of glomerular disease in Col4a3 KO mice. Instead, TSP1 was robustly up-regulated in tubules and interstitium within fibrotic kidneys of Col4a3 KO mice (Figure 10A). Tubular atrophy, determined by masked scoring of the percentage of atrophic tubules among all tubules, was more pronounced in the Col4a3 $\mathrm{KO}$ mice (Figure 10B). The growth factor TGF- $\beta 1$ is a principal mediator of progression of CKD. ${ }^{1,34}$ To gain insight into the role of TSP1 on TGF- $\beta 1$ activation and progression of CKD in Col4a3 KO mice, we performed immunostaining with antibodies to phosphorylated SMADs 2 and 3 (p-SMAD2/3), which detects active TGF- $\beta 1$ signaling. ${ }^{35}$ Consistent with the robust up-regulation of TSP1 observed in tubules and interstitium of fibrotic kidneys of Col4a3 KO mice, tubular and interstitial p-SMAD2/3 was markedly increased (Figure 10, C and D). Compared with Col4a3 KO mice (increased TSP1), tubular and interstitial immunostaining of p-SMAD2/3 was attenuated in creatinine-matched Col4a3;Tspl DKO mice (Figure 10, C and D).

\section{Proliferation and Collagen Expression in Response to Latent TGF- $\beta 1$ Is Blunted in TSP1-Deficient Fibroblasts}

Previous studies have demonstrated that TSP1 activates latent TGF- $\beta 1 .^{36}$ To determine whether altered disease progression in the absence of TSP1 is due to failure to activate latent TGF- $\beta 1$, we next explored the capacity of TSP1-deficient cells to respond to latent TGF- $\beta 1$ in vitro. Because fibroblast accumulation and ECM deposition were blunted in kidneys of end-stage diseased Col4a3; Tspl DKO mice (at 32 weeks), compared with the Col4a3 KO counterparts (at 22 weeks), and because TGF- $\beta 1$ is known to induce both proliferation and ECM synthesis in fibroblasts, we focused on renal fibroblasts. We isolated primary fibroblasts from kidneys of Tspl $\mathrm{KO}$ mice and C57BL/6 control mice and compared their proliferation and procollagen III expression in response to either latent or active TGF- $\beta 1$. Although the proliferative response of Tspl KO fibroblasts to latent TGF- $\beta 1$ was significantly decreased, compared with wild-type C57BL/6 fibroblasts, their response to active TGF- $\beta 1$ was equal (Figure 11A).
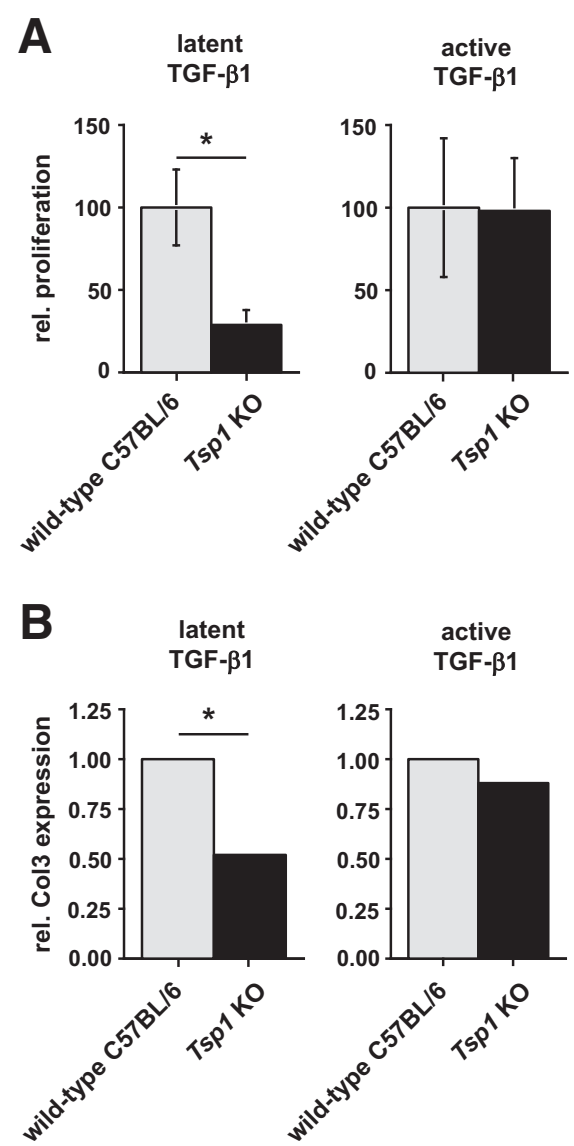

Figure 11 TSP1 deficiency causes an altered response to latent TGF- $\beta 1$. A: Fibroblast proliferation in response to active and latent TGF- $\beta 1$. Primary fibroblasts isolated from kidneys of wild-type control and TSP1-deficient mice (Tsp1 K0) were exposed for 24 hours to $5 \mathrm{ng} / \mathrm{mL}$ of either latent TGF- $\beta 1$ or active TGF- $\beta 1$. Relative proliferation was assessed by a WST assay. TSP1 deficiency significantly impaired the proliferative response to latent TGF- $\beta 1$, but not to active TGF- $\beta 1$. B: Type III collagen expression in response to active and latent TGF- $\beta 1$. Wild-type control and Tsp1 K0 renal fibroblasts were exposed for 24 hours to $5 \mathrm{ng} / \mathrm{mL}$ of either latent TGF- $\beta 1$ or active TGF- $\beta 1$. Type III procollagen expression was assessed by quantitative real-time PCR. TSP1 deficiency significantly decreased collagen III expression in response to latent TGF- $\beta 1$. Data are expressed as means \pm SEM. ${ }^{*} P<0.05$. Col3, collagen III; rel., relative. 
Similarly, collagen III expression was blunted in response to latent TGF- $\beta 1$ but not to active TGF- $\beta 1$ (Figure 11B). In summary, these findings suggest that ineffective activation of latent TGF- $\beta 1$ is likely the underlying cause for altered kidney disease progression in Col4a3;Tspl DKO mice.

\section{Discussion}

We conducted the present study to gain mechanistic insight into the role of TSP1 in the progression of CKD. For this purpose, we compared kidney disease progression in Col4a3 KO mice with that of Col4a3;Tsp1 DKO mice. Decline of excretory renal function was delayed by the absence of TSP1, confirming previous reports. ${ }^{9-13}$ Unexpectedly, comparison of creatinine-matched kidneys of Col4a3 KO and Col4a3;Tsp1 DKO mice revealed that disease progression was not simply delayed, but that kidney disease followed different paths. Despite similar interstitial volumes at matched creatinine levels, absence of TSP1 in Col4a3;Tspl DKO mice was associated with lesser collagen deposition, lesser fibroblast accumulation, and lesser tubular atrophy, compared with Col4a3 KO mice, but increased Th1-mediated inflammation. The extent of glomerulosclerosis and the density of microvessels were not altered by the absence of TSP1. Our findings suggest that the altered course of disease progression in Col4a3;Tsp1 DKO mice is caused by an inability of kidney cells to activate latent TGF- $\beta 1$ in the absence of TSP1. Our findings are consistent with the literature on the role of TSP1 in TGF- $\beta 1$ activation. TGF- $\beta 1$ is known to induce fibroblast proliferation and collagen deposition, and to contribute to formation of tubular atrophy, all of which are reduced in the absence of TGF- $\beta 1$-activating TSP1. ${ }^{1,37}$ TGF- $\beta 1$-deficient mice exhibit severe inflammatory lesions, and TGF- $\beta 1$ in general is known to be anti-inflammatory. ${ }^{38,39}$ In the present study, inability to activate latent TGF- $\beta 1$ was associated with an increased inflammatory response in chronic progressive kidney disease. With regard to progression of CKD, it is an unexpected finding that decline of kidney function is caused by different pathways, depending on the presence or absence of TSP1. Our findings suggest that fibroproliferative and inflammatory responses are distinct entities, both of which (and possibly independent of each another) can determine progression of CKD. Our present findings confirm earlier findings indicating that relative interstitial volume is the most accurate correlate of chronic progression of kidney disease, but challenge the concept of one common uniform pathway leading to ESRF.

Additionally, our findings suggest that a fibrosisdominated pathology is more detrimental for kidney function than is inflammation-dominated response, but that either pathological entity can ultimately cause decline of kidney function. Although the common denominator appears to be increased interstitial volume, it remains unclear how such expansion of the interstitium causes decline in kidney function. We speculate that both fibrosis and inflammation can cause vascular and/or tubular occlusion through volume expansion. The present findings give reason for caution, and suggest that future therapeutic strategies exclusively targeting fibrosis could be less effective than combined inhibition of fibrosis and inflammation. The concept of a uniform pathway of chronic progressive kidney disease may require further consideration, and fibroproliferative and inflammatory components of interstitial disease may need to be viewed as separate causal entities.

\section{Acknowledgments}

We thank Sai Ram Reddy, Scott McGoohan, Lauren Orefice, and Margot Martino for technical assistance.

\section{References}

1. Zeisberg M, Neilson EG: Mechanisms of tubulointerstitial fibrosis. J Am Soc Nephrol 2010, 21:1819-1834

2. McClellan AC, Plantinga L, McClellan WM: Epidemiology, geography and chronic kidney disease. Curr Opin Nephrol Hypertens 2012, 21:323-328

3. Zeisberg M, Kalluri R: Experimental strategies to reverse chronic renal disease. Blood Purif 2004, 22:440-445

4. Risdon RA, Sloper JC, De Wardener HE: Relationship between renal function and histological changes found in renal-biopsy specimens from patients with persistent glomerular nephritis. Lancet 1968, 2: 363-366

5. Bohle A, Bader R, Grund KE, Mackensen S, Neunhoeffer: Serum creatinine concentration and renal interstitial volume. Analysis of correlations in endocapillary (acute) glomerulonephritis and in moderately severe mesangioproliferative glomerulonephritis. Virchows Arch A Pathol Anat Histol 1977, 375:87-96

6. Bohle A, Glomb D, Grund KE, Mackensen S: Correlation between relative interstitial volume of the renal cortex and serum creatinine concentration in minimal changes with nephrotic syndrome and in focal sclerosing glomerulonephritis. Virchows Arch A Pathol Anat Histol 1977, 376:221-232

7. Bohle A, Wehrmann M, Bogenschutz O, Batz C, Müller CA, Müller GA: The pathogenesis of chronic renal failure in diabetic nephropathy. Investigation of 488 cases of diabetic glomerulosclerosis. Pathol Res Pract 1991, 187:251-259

8. Armstrong LC, Bornstein P: Thrombospondins 1 and 2 function as inhibitors of angiogenesis. Matrix Biol 2003, 22:63-71

9. Daniel C, Wiede J, Krutzsch HC, Ribeiro SM, Roberts DD, MurphyUllrich JE, Hugo C: Thrombospondin-1 is a major activator of TGFbeta in fibrotic renal disease in the rat in vivo. Kidney Int 2004, 65: 459-468

10. Lu A, Miao M, Schoeb TR, Agarwal A, Murphy-Ullrich JE: Blockade of TSP1-dependent TGF- $\beta$ activity reduces renal injury and proteinuria in a murine model of diabetic nephropathy. Am J Pathol 2011, 178: $2573-2586$

11. Bige N, Shweke N, Benhassine S, Jouanneau C, Vandermeersch S, Dussaule JC, Chatziantoniou C, Ronco P, Boffa JJ: Thrombospondin-1 plays a profibrotic and pro-inflammatory role during ureteric obstruction. Kidney Int 2012, 81:1226-1238

12. Daniel C, Takabatake Y, Mizui M, Isaka Y, Kawashi H, Rupprecht H, Imai E, Hugo C: Antisense oligonucleotides against thrombospondin-1 inhibit activation of TGF-beta in fibrotic renal disease in the rat in vivo. Am J Pathol 2003, 163:1185-1192 
13. Hochegger K, Knight S, Hugo C, Mayer G, Lawler J, Mayadas TN, Rosenkranz AR: Role of thrombospondin-1 in the autologous phase of an accelerated model of anti-glomerular basement membrane glomerulonephritis. Nephron Exp Nephrol 2004, 96:e31-e38

14. Chen H, Herndon ME, Lawler J: The cell biology of thrombospondin-1. Matrix Biol 2000, 19:597-614

15. Xie L, Duncan MB, Pahler J, Sugimoto H, Martino M, Lively J, Mundel T, Soubasakos M, Rubin K, Takeda T, Inoue M, Lawler J, Hynes RO, Hanahan D, Kalluri R: Counterbalancing angiogenic regulatory factors control the rate of cancer progression and survival in a stage-specific manner. Proc Natl Acad Sci USA 2011, 108: 9939-9944

16. Sugimoto H, Mundel TM, Sund M, Xie L, Cosgrove D, Kalluri R: Bone-marrow-derived stem cells repair basement membrane collagen defects and reverse genetic kidney disease. Proc Natl Acad Sci USA 2006, 103:7321-7326

17. Hamano Y, Sugimoto H, Soubasakos MA, Kieran M, Olsen BR, Lawler J, Sudhakar A, Kalluri R: Thrombospondin-1 associated with tumor microenvironment contributes to low-dose cyclophosphamidemediated endothelial cell apoptosis and tumor growth suppression. Cancer Res 2004, 64:1570-1574

18. Miner JH, Sanes JR: Molecular and functional defects in kidneys of mice lacking collagen alpha 3(IV): implications for Alport syndrome. J Cell Biol 1996, 135:1403-1413

19. Zeisberg M, Bottiglio C, Kumar N, Maeshima Y, Strutz F, Müller GA, Kalluri R: Bone morphogenic protein-7 inhibits progression of chronic renal fibrosis associated with two genetic mouse models. Am J Physiol Renal Physiol 2003, 285:F1060-F1067

20. Zeisberg EM, Potenta SE, Sugimoto H, Zeisberg M, Kalluri R: Fibroblasts in kidney fibrosis emerge via endothelial-to-mesenchymal transition. J Am Soc Nephrol 2008, 19:2282-2287

21. Zeisberg EM, Tarnavski O, Zeisberg M, Dorfman AL, McMullen JR, Gustafsson E, Chandraker A, Yuan X, Pu WT, Roberts AB, Neilson EG, Sayegh MH, Izumo S, Kalluri R: Endothelial-to-mesenchymal transition contributes to cardiac fibrosis. Nat Med 2007, 13:952-961

22. Okuno Y, Huettner CS, Radomska HS, Petkova V, Iwasaki H, Akashi K, Tenen DG: Distal elements are critical for human CD34 expression in vivo. Blood 2002, 100:4420-4426

23. Zeisberg M, Khurana M, Rao VH, Cosgrove D, Rougier JP, Werner MC, Shield CF 3rd, Werb Z, Kalluri R: Stage-specific action of matrix metalloproteinases influences progressive hereditary kidney disease. PLoS Med 2006, 3:e100

24. Sugimoto H, LeBleu VS, Bosukonda D, Keck P, Taduri G, Bechtel W, Okada H, Carlson W Jr, Bey P, Rusckowski M, Tampe B, Tampe D, Kanasaki K, Zeisberg M, Kalluri R: Activin-like kinase 3 is important for kidney regeneration and reversal of fibrosis. Nat Med 2012, 18:396-404

25. Hoffmann KF, McCarty TC, Segal DH, Chiaramonte M, Hesse M, Davis EM, Cheever AW, Meltzer PS, Morse HC 3rd, Wynn TA:
Disease fingerprinting with cDNA microarrays reveals distinct gene expression profiles in lethal type 1 and type 2 cytokine-mediated inflammatory reactions. FASEB J 2001, 15:2545-2547

26. Liu L, Kou P, Zeng Q, Pei G, Li Y, Liang H, Xu G, Chen S: CD4+ T lymphocytes, especially Th2 cells, contribute to the progress of renal fibrosis. Am J Nephrol 2012, 36:386-396

27. Sandler NG, Mentink-Kane MM, Cheever AW, Wynn TA: Global gene expression profiles during acute pathogen-induced pulmonary inflammation reveal divergent roles for Th1 and Th2 responses in tissue repair. J Immunol 2003, 171:3655-3667

28. Wynn TA: Fibrotic disease and the $\mathrm{T}(\mathrm{H}) 1 / \mathrm{T}(\mathrm{H}) 2$ paradigm. Nat Rev Immunol 2004, 4:583-594

29. Zhang HP, Wu Y, Liu J, Jiang J, Geng XR, Yang G, Mo L, Liu ZQ, Liu ZG, Yang PC: TSP1-producing B cells show immune regulatory property and suppress allergy-related mucosal inflammation. Sci Rep 2013, 3:3345

30. Yang K, Vega JL, Hadzipasic M, Schatzmann Peron JP, Zhu B, Carrier Y, Masli S, Rizzo LV, Weiner HL: Deficiency of thrombospondin-1 reduces Th17 differentiation and attenuates experimental autoimmune encephalomyelitis. J Autoimmun 2009, 32:94-103

31. Fu S, Zhang N, Yopp AC, Chen D, Mao M, Zhang H, Ding Y, Bromberg JS: TGF-beta induces Foxp3+ T-regulatory cells from CD4+ CD25 - precursors. Am J Transplant 2004, 4:1614-1627

32. Gandolfo MT, Jang HR, Bagnasco SM, Ko GJ, Agreda P, Satpute SR, Crow MT, King LS, Rabb H: Foxp3+ regulatory T cells participate in repair of ischemic acute kidney injury. Kidney Int 2009, 76:717-729

33. Kinsey GR, Huang L, Vergis AL, Li L, Okusa MD: Regulatory T cells contribute to the protective effect of ischemic preconditioning in the kidney. Kidney Int 2010, 77:771-780

34. Wynn TA: Fibrosis under arrest. Nat Med 2010, 16:523-525

35. Zeisberg M, Hanai J, Sugimoto H, Mammoto T, Charytan D, Strutz F, Kalluri R: BMP-7 counteracts TGF-beta1-induced epithelial-tomesenchymal transition and reverses chronic renal injury. Nat Med 2003, 9:964-968

36. Schultz-Cherry S, Lawler J, Murphy-Ullrich JE: The type 1 repeats of thrombospondin 1 activate latent transforming growth factor-beta. J Biol Chem 1994, 269:26783-26788

37. Bechtel W, McGoohan S, Zeisberg EM, Müller GA, Kalbacher H, Salant DJ, Müller CA, Kalluri R, Zeisberg M: Methylation determines fibroblast activation and fibrogenesis in the kidney. Nat Med 2010, 16: $544-550$

38. Christ M, McCartney-Francis NL, Kulkarni AB, Ward JM, Mizel DE, Mackall CL, Gress RE, Hines KL, Tian H, Karlsson S, Wahl SM: Immune dysregulation in TGF-beta 1-deficient mice. J Immunol 1994, $153: 1936-1946$

39. Kulkarni AB, Karlsson S: Inflammation and TGF beta 1: lessons from the TGF beta 1 null mouse. Res Immunol 1997, 148:453-456 